\title{
Special Functions in Minimal Representations
}

\author{
Toshiyuki Kobayashi \\ Dedicated to Igor Frenkel on the occasion of his 60th birthday with great admire

\begin{abstract}
Minimal representations of a real reductive group $G$ are the 'smallest' irreducible unitary representations of $G$. We discuss special functions that arise in the analysis of $L^{2}$-model of minimal representations.
\end{abstract}

\section{Introduction}

An irreducible unitary representation of a real reductive Lie group $G$ is called minimal if its infinitesimal representation is annihilated by the Joseph ideal [11] in the enveloping algebra. Loosely speaking, minimal representations of $G$ are the 'smallest' infinite dimensional unitary representations.

The Weil representation, known for a prominent role in number theory (e.g. the theta correspondence), provides minimal representations of the metaplectic group $M p(n, \mathbb{R})$. The minimal representation of a conformal group $S O(4,2)$ appears in mathematical physics, e.g., as the bound states of the hydrogen atom, and incidentally as the quantum Kepler problem. In these classical examples the representations are highest weight modules, however, for more general reductive groups, minimal representations (if exist) may not be highest weight modules, see a pioneering work of Kostant [21] for $S O(4,4)$.

In the last decade I have been developing a geometric and analytic theory of minimal representations with my collaborators, S. Ben Saïd, J. Hilgert, G. Mano, J. Möllers, B. Ørsted, and M. Pevzner, see $[1,6,7,8,9,12,13,15,16,17,18,19$, 20]. Among all, in this paper, we focus on 'special functions' that arise naturally in the $L^{2}$-model of minimal representations. Needless to say, the interaction between special functions and group representations has a long history and there is extensive literature on this subject. A new feature in our setting for minimal representations is that the representation of the group is realized on the Hilbert space $L^{2}(\Xi)$ where the dimension of a manifold $\Xi$ (see (2.1), or more generally Section 2.3 for the definition of $\Xi$ ) is strictly smaller than the dimension of any nontrivial $G$-space in most cases. This means that $G$ cannot act geometrically on $\Xi$ but there is

2010 Mathematics Subject Classification. Primary 22E30; Secondary 22E46, 33C45.

The author was partially supported by Grant-in-Aid for Scientific Research (B) (22340026), Japan Society for the Promotion of Sciences.

(C)0000 (copyright holder) 
a natural action of $G$ on $L^{2}(\Xi)$. As a result, the Casimir element of a compact subgroup acts as a fourth-order differential operator. The indefinite orthogonal group $G=O(p+1, q+1)$ is the most interesting for this purpose in the sense that the group $G$ itself contains two parameters $p$ and $q$, and we shall highlight this case by giving occasionally some perspectives to other reductive groups.

Acknowledgements: I first met Igor Frenkel when I visited Yale University in 2009 to give a colloquium talk. It was then a surprising pleasure that Igor told me his recent theory on quaternionic analysis $[\mathbf{3}, \mathbf{4}, \mathbf{5}]$ with Libine uses some of my work $[\mathbf{1 7}, \mathbf{1 9}]$ on geometric analysis of minimal representations, which encourages me to develop further the analytic theory of minimal representations.

I would like to thank the organizers P. Etingof, M. Khovanov, A. Kirillov Jr., A. Lachowska, A. Licata, A. Savage and G. Zuckerman for their hospitality during the stimulating conference "Perspectives in Representation Theory" in honor of Prof. Igor Frenkel's 60th birthday at Yale University, 12-16 May 2012. Thanks are also due to an anonymous referee for his/her careful comments.

\section{A generalization of the Fourier transform}

2.1. Algebraic characterization of Fourier transforms. We begin with an algebraic characterization of the Euclidean Fourier transform $\mathcal{F}_{\mathbb{R}^{n}}$. Let $Q_{j}:=x_{j}$ be the multiplication operators by coordinates, and $P_{j}:=\frac{1}{\sqrt{-1}} \frac{\partial}{\partial x_{j}}$. Then we have:

Proposition 2.1. Any continuous operator $A$ on $L^{2}\left(\mathbb{R}^{n}\right)$ satisfying

$$
A \circ Q_{j}=P_{j} \circ A, \quad A \circ P_{j}=-Q_{j} \circ A \quad \text { on } \mathcal{S}\left(\mathbb{R}^{n}\right) \quad(1 \leq j \leq n)
$$

is a scalar multiple of the Euclidean Fourier transform $\mathcal{F}_{\mathbb{R}^{n}}$. In particular, any such continuous operator $A$ is unitary up to scaling.

In place of $\mathbb{R}^{n}$, let us consider the isotropic cone

$$
\Xi:=\left\{x \in \mathbb{R}^{p+q} \backslash\{0\}: x_{1}^{2}+\cdots+x_{p}^{2}-x_{p+1}^{2}-\cdots-x_{p+q}^{2}=0\right\},
$$

equipped with a measure $d \mu=\frac{1}{2} r^{p+q-3} d r d \omega d \eta$ in the bipolar coordinates:

$$
\mathbb{R}_{+} \times S^{p-1} \times S^{q-1} \stackrel{\sim}{\rightarrow} \Xi, \quad(r, \omega, \eta) \mapsto(r \omega, r \eta) .
$$

REMARK 2.2. This cone $\Xi$ is a special case of the Lagrangian submanifold of a minimal real coadjoint orbit, denoted by the same letter $\Xi$, given in Theorem 2.10 where we deal with more general reductive groups.

Recall from [17] that the fundamental differential operators $R_{j}(1 \leq j \leq p+q)$ on $\Xi$ are mutually commuting operators which are obtained as the restriction of the tangential differential operators

$$
\varepsilon_{j} x_{j} \square-(2 E+p+q-2) \frac{\partial}{\partial x_{j}}
$$

to $\Xi$, where $\varepsilon_{j}=1(1 \leq j \leq p)$; $=-1(p+1 \leq j \leq p+q), \square:=\sum_{a=1}^{p+q} \varepsilon_{a} \frac{\partial^{2}}{\partial x_{a}^{2}}$ (the Laplacian on $\mathbb{R}^{p, q}$ ) and $E:=\sum_{a=1}^{p+q} x_{a} \frac{\partial}{\partial x_{a}}$ (the Euler operator). Then we have

Theorem 2.3 ([17, Theorem 1.2.3]). Suppose $p+q$ is even, $\geq 4$. Then there exists a unitary operator $\mathcal{F}_{\Xi}$ on $L^{2}(\Xi)$ satisfying the following relation for $A$ :

$$
A \circ Q_{j}=R_{j} \circ A, \quad A \circ R_{j}=Q_{j} \circ A \quad \text { on } C_{c}^{\infty}(\Xi) \quad(1 \leq j \leq p+q) .
$$


Conversely, any continuous operator A satisfying (2.3) is a scalar multiple of the unitary operator $\mathcal{F}_{\Xi}$.

2.2. Unitary inversion operator $\mathcal{F}_{\Xi}$. The similar nature of $\mathcal{F}_{\mathbb{R}^{n}}$ and $\mathcal{F}_{\Xi}$ indicated in Proposition 2.1 and Theorem 2.3 is derived from the common fact that they arise as the unitary inversion operators in the $L^{2}$-model (Schrödinger model) of minimal representations of real reductive groups $M p(n, \mathbb{R})$ and $O(p+1, q+1)$, respectively.

To see some more details of Theorem 2.3 , let $\overline{\mathfrak{n}}, \mathfrak{n}$, and $\mathfrak{l}$ be the Lie algebras generated by the operators $Q_{i}, R_{j}$, and $\left[Q_{i}, R_{j}\right](1 \leq i, j \leq p+q)$. Then $\mathfrak{g}:=\overline{\mathfrak{n}}+\mathfrak{l}+\mathfrak{n}$ is isomorphic to $\mathfrak{o}(p+1, q+1)$, and $\mathfrak{p}=\mathfrak{l}+\mathfrak{n} \simeq(\mathfrak{o}(p, q)+\mathbb{R}) \ltimes \mathbb{R}^{p+q}$ is a maximal parabolic subalgebra of $\mathfrak{g}$.

For $p+q$ even, $\geq 4$, we proved in [19] that there exists an irreducible unitary representation $\pi$ of the group $G:=O(p+1, q+1)$ on the Hilbert space $L^{2}(\Xi)$ of which the infinitesimal representation is given by $Q_{j}$ (the action of $\mathfrak{n}$ ) and $R_{j}$ (the action of $\overline{\mathfrak{n}})$, see also $[\mathbf{1 7}$, Chapter 1$]$. We set

$$
w:=\left(\begin{array}{cc}
I_{p} & O \\
O & -I_{q}
\end{array}\right) \in G
$$

Geometrically, $\mathfrak{p}$ is the Lie algebra of the conformal transformation group $(O(p, q)$. $\left.\mathbb{R}_{>0}\right) \ltimes \mathbb{R}^{p, q}$ of the flat pseudo-Riemannian Euclidean space $\mathbb{R}^{p, q}$, and $w$ induces the conformal inversion of $\mathbb{R}^{p, q}$ by the Möbius transform.

The unitary operators $\pi(g)$ are of simple form if $g \in G$ belong to the maximal parabolic subgroup $P$ with Lie algebra $\mathfrak{p}$, namely, they are given by the multiplication of certain elementary functions on $\Xi$ and the translations coming from the geometric action of the Levi subgroup of $P$ on $\Xi$ (see [17, Chap.2, Sect.3]). In view of the Bruhat decomposition

$$
G=P \amalg P w P,
$$

it is enough to find an explicit formula of the unitary operator $\pi(w)$ in order to give a global formula of the $G$-action on $L^{2}(\Xi)$. We call $\pi(w)$ the unitary inversion operator, and set

$$
\mathcal{F}_{\Xi}:=\pi(w) .
$$

We initiated in a series of papers $[\mathbf{1 5}, \mathbf{1 6}, \mathbf{1 7}]$ the following:

Program 2.4 ([17, Program 1.2.5]). Use the unitary inversion operator $\mathcal{F}_{\Xi}$ for minimal representations as an analog of the Euclidean Fourier transform $\mathcal{F}_{\mathbb{R}^{n}}$, and develop a theory of 'Fourier analysis' on $\Xi$.

In the classical Schrödinger model of the Weil representation of the metaplectic group $M p(n, \mathbb{R})$ on $L^{2}\left(\mathbb{R}^{n}\right)$, the unitary inversion operator is nothing but the Euclidean Fourier transform $\mathcal{F}_{\mathbb{R}^{n}}$ (up to scalar of modulus one), see Example 3.1. We note that $M p(n, \mathbb{R})$ and $O(p+1, q+1)$ with $p+q$ even are simple Lie groups of type $C$ and $D$, respectively.

The first stage of Program 2.4 is to establish a framework of the $L^{2}$-model (Schrödinger model) of minimal representations, and to introduce the unitary inversion operator $\mathcal{F}_{\Xi}$ with an algebraic characterization such as Theorem 2.3. In [8] we gave such a model and defined $\mathcal{F}_{\Xi}$ by using Jordan algebras, see Section 2.3. In this case $\Xi$ is a Lagrangian subvariety of a minimal nilpotent coadjoint orbit and the resulting representations on $L^{2}(\Xi)$ include a slightly wider family of unitary 
representations than minimal representations (e.g. the full complementary series representations of $O(n, 1))$.

The second stage is to solve the following:

Problem 2.5. Find an explicit formula of the integral kernel of $\mathcal{F}_{\Xi}$.

We will discuss Problem 2.5 in Section 3. It is noteworthy that I. Frenkel and M. Libine have developed their original theory on quaterionic analysis in a series of papers $[\mathbf{3}, \mathbf{4}, \mathbf{5}]$ from the viewpoint of representation theory of the conformal group $S L\left(2, \mathbb{H}_{\mathbb{C}}\right) \simeq S L(4, \mathbb{C})$ and its real forms, and have demonstrated a close connection between minimal representations of various $O(p, q)$ 's and quaternionic analysis. For instance, the explicit formula of $\mathcal{F}_{\Xi}$ for $O(3,3)$ which was obtained in Kobayashi-Mano [17] is used in [5] for the study of the key operator $\left(P l_{R}\right.$ in their notation) in the analysis of the space $\mathbb{H}_{\mathbb{R}}$ of split quaternions.

The third stage and beyond will be based on the algebraic property (Theorem 2.3) and analytic property (Problem 2.5) of the unitary inversion operator $\mathcal{F}_{\Xi}$. Among various, potentially interesting directions of the 'Fourier analysis' on $\Xi$, here are some few topics:

- A theory of holomorphic semigroups was given in Howe [10] for $M p(n, \mathbb{R})$ and in Kobayashi-Mano [16] for $S O(n+1,2)$.

- A deformation theory of the Euclidean Fourier transform $\mathcal{F}_{\mathbb{R}^{n}}[\mathbf{1}]$, e.g. an interpolation between $\mathcal{F}_{\mathbb{R}^{n}}$ and the unitary inversion operator of $S O(n+$ $1,2)$.

- A generalization of the classical Bargmann-Segal transform. See [9] in the case $G / K$ is of tube type.

Stage 1 already includes a solution for the Plancherel-type theorem of $\mathcal{F}_{\Xi}$. A natural but open question would be a Paley-Wiener type theorem of $\mathcal{F}_{\Xi}$ :

QUESTION 2.6. Find an explicit characterization of $\mathcal{F}_{\Xi}\left(C_{c}^{\infty}(\Xi)\right)$.

Another important space of functions is an analog of Schwartz functions. For this we may consider:

Definition 2.7 (Schwartz space on $\Xi$ ). Let $\mathcal{S}(\Xi)$ be the Fréchet space of smooth vectors of the unitary representation of $G$ on $L^{2}(\Xi)$.

This definition makes sense in a more general setting (see Theorem 2.10). By the general theory of unitary representations, we have:

Proposition 2.8. $\mathcal{F}_{\Xi}$ induces automorphisms of the Hilbert space $L^{2}(\Xi)$ and the Fréchet space $\mathcal{S}(\Xi)$.

$$
\begin{aligned}
& \mathcal{F}_{\Xi}: L^{2}(\Xi) \stackrel{\sim}{\rightarrow} L^{2}(\Xi) \quad \text { (Plancherel type theorem) }, \\
& \cup \quad \cup \\
& \mathcal{S}(\Xi) \stackrel{\sim}{\rightarrow} \mathcal{S}(\Xi) \quad \text { (Paley-Wiener type theorem }) .
\end{aligned}
$$

The following question is also open:

QUESTION 2.9. Find an explicit characterization of $\mathcal{S}(\Xi)$. 
2.3. Schrödinger model of minimal representations. Suppose that $V$ is a real simple Jordan algebra. Let $G$ and $L$ be the identity components of the conformal group and the structure group of the Jordan algebra $V$, respectively. Then the Lie algebra $\mathfrak{g}$ is a real simple Lie algebra and has a Gelfand-Naimark decomposition $\mathfrak{g}=\overline{\mathfrak{n}}+\mathfrak{l}+\mathfrak{n}$, where $\mathfrak{n} \simeq V$ is regarded as an Abelian Lie algebra, $\mathfrak{l} \simeq \mathfrak{s t r}(V)$ the structure algebra, and $\overline{\mathfrak{n}}$ acts on $V$ by quadratic vector fields.

Let $\mathbb{O}_{\min }^{G_{\mathbb{R}}}\left(\subset \mathfrak{g}^{*}\right)$ be a (real) minimal nilpotent coadjoint orbit. By identifying $\mathfrak{g}$ with the dual $\mathfrak{g}^{*}$, we consider the intersection

$$
\Xi:=V \cap \mathbb{O}_{\text {min }}^{G_{\mathbb{R}}},
$$

which is a Lagrangian submanifold of the symplectic manifold $\mathbb{O}_{\min }^{G_{\mathbb{R}}}$ endowed with the Kirillov-Kostant-Souriau symplectic form. There is a natural $L$-invariant Radon measure on $\Xi$, and we write $L^{2}(\Xi)$ for the Hilbert space consisting of square integrable functions on $\Xi$.

TheOREM 2.10 (Schrödinger model $[\mathbf{8}]$ ). Suppose $V$ is a real simple Jordan algebra such that its maximal Euclidean Jordan algebra is also simple. Among all such Jordan algebras $V$, we exclude the case where $V \simeq \mathbb{R}^{p, q}$ with $p+q$ odd (see Examples 2.13 and 2.14).

1) For an appropriate finite covering group $\widetilde{G}$ of $G$ there exists a natural unitary representation of $\widetilde{G}$ on $L^{2}(\Xi)$. It is irreducible if and only if $\Xi$ is connected.

2) The Gelfand-Kirillov dimension of $\pi$ attains its minimum among all infinite dimensional representations of $\widetilde{G}$, i.e. $\operatorname{DIM}(\pi)=\frac{1}{2} \operatorname{dim} \mathbb{O}_{\text {min }}^{G_{\mathbb{R}}}$.

3) The annihilator of the differential representation $d \pi$ is the Joseph ideal in the enveloping algebra $U\left(\mathfrak{g}_{\mathbb{C}}\right)$ if $V$ is split and $\mathfrak{g}_{\mathbb{C}}$ is not of type $A$.

The simple Lie algebras $\mathfrak{g}$ that appear in Theorem 2.10 are categorized into four cases as below:

$$
\begin{aligned}
& \mathfrak{s l}(2 k, \mathbb{R}), \mathfrak{s o}(2 k, 2 k), \mathfrak{s o}(p+1, q+1), \mathfrak{e}_{7(7)}, \\
& \mathfrak{s p}(k, \mathbb{R}), \mathfrak{s u}(k, k), \mathfrak{s o}{ }^{*}(4 k), \mathfrak{s o}(2, k), \mathfrak{e}_{7(-25)}, \\
& \mathfrak{s p}(k, \mathbb{C}), \mathfrak{s l}(2 k, \mathbb{C}), \mathfrak{s o}(4 k, \mathbb{C}), \mathfrak{s o}(k+2, \mathbb{C}), \mathfrak{e}_{7}(\mathbb{C}), \\
& \mathfrak{s p}(k, k), \mathfrak{s u}{ }^{*}(4 k), \mathfrak{s o}(k, 1) .
\end{aligned}
$$

EXAmPLE 2.11. If $V$ is a Euclidean Jordan algebra, then $G$ is the automorphism group of a Hermitian symmetric space of tube type and the corresponding Lie algebra $\mathfrak{g}$ is listed in (2.7). In this case $\Xi$ consists of two connected components, and the resulting representation $\pi$ is the direct sum of an irreducible unitary highest weight module and its dual.

REMARK 2.12. In the case (2.9) the complex minimal nilpotent orbit $\mathbb{O}_{\text {min }}^{G_{\complement}}$ does not meet the real form $\mathfrak{g}$, and there does not exist an admissible representation of any Lie group with Lie algebra $\mathfrak{g}$. In particular, the representation $\pi$ in Theorem 2.10 is not a minimal representation but still one of the 'smallest' infinite dimensional representations in the sense that the Gelfand-Kirillov dimension attains its minimum.

EXAMPle 2.13. Let $V=\operatorname{Sym}(n, \mathbb{R})$. Then $\mathfrak{g}=\mathfrak{s p}(n, \mathbb{R})$ and

$$
\Xi=\left\{X \in M(n, \mathbb{R}): X={ }^{t} X, \operatorname{rank} X=1\right\} .
$$


Let $\Xi_{+}:=\left\{X \in V \cap \mathbb{O}_{\text {min }}^{G_{\mathbb{R}}}\right.$ : Trace $\left.X>0\right\}$. Via the double covering map (folding map)

$$
\mathbb{R}^{n} \backslash\{0\} \rightarrow \Xi_{+}, \quad v \mapsto v^{t} v
$$

we can identify the representation on $L^{2}\left(\Xi_{+}\right)$with the even part of the Schrödinger model on $L^{2}\left(\mathbb{R}^{n}\right)$ of the metaplectic group $M p(n, \mathbb{R})[\mathbf{2}, \mathbf{1 0}]$. See $[\mathbf{8}]$ for the realization of the odd part of the Weil representation in the space of sections for a certain line bundle over $\Xi_{+}$.

EXAMPLE 2.14. We define a multiplication on $\mathbb{R}^{p+q}=\mathbb{R} \oplus \mathbb{R}^{p+q-1}$ by

$$
\left(x_{1}, x^{\prime}\right) \cdot\left(y_{1}, y^{\prime}\right):=\left(x_{1} y_{1}-\sum_{i=2}^{p} x_{i} y_{i}+\sum_{i=p+1}^{p+q} x_{i} y_{i}, x_{1} y^{\prime}+y_{1} x^{\prime}\right) .
$$

The resulting Jordan algebra is denoted by $\mathbb{R}^{p, q}$ (by a little abuse of notation). It is a semisimple Jordan algebra of rank two, and its conformal algebra is $\mathfrak{o}(p+1, q+1)$. Suppose now $V=\mathbb{R}^{p, q}$ with $p+q$ even. Then $\Xi$ in Theorem 2.10 coincides with the isotropic cone given in (2.1). For $q=1, V$ is an Euclidean Jordan algebra, and $\Xi$ consists of two connected components according to the sign of the first coordinate $x_{1}$, i.e. the past and future cones. For $p, q \geq 2, V$ is non-Euclidean, $\Xi$ is connected, and our representation $\pi$ on $L^{2}(\Xi)$ is the same as the Schrödinger model of the minimal representation of $O(p+1, q+1)$ constructed in [19, Part III], which is neither a highest nor a lowest weight module.

REMARK 2.15. There is no minimal representation for any group with Lie algebra $\mathfrak{o}(p+1, q+1)$ with $p+q$ odd, $p, q \geq 3$ (see [23, Theorem 2.13]).

\section{Unitary inversion operator $\mathcal{F}_{\Xi}$}

By the Schwartz kernel theorem, the unitary inversion operator $\mathcal{F}_{\Xi}$ can be given by a distribution kernel $K(x, y) \in \mathcal{D}^{\prime}(\Xi \times \Xi)$, namely,

$$
\mathcal{F}_{\Xi} u(x)=\int_{\Xi} K(x, y) u(y) d \mu(y) \quad \text { for all } u \in C_{c}^{\infty}(\Xi) .
$$

Problem 2.5 asks for an explicit formula of $K(x, y)$. In the setting of Theorem 2.10, we can generalize the definition (2.5) of $\mathcal{F}_{\Xi}$ by taking $w$ to be a lift of the conformal inversion on $V$, see [9]. So far, Problem 2.5 has been solved for minimal representations in the following two cases:

Case A. $G$ is the biholomorphic transformation group of a Hermitian symmetric space of tube type $([\mathbf{9}])$.

Case B. $G=O(p+1, q+1)$ (Theorem 3.3).

Case A includes the following earlier results:

ExAmple 3.1. $\quad$ 1) $G=M p(n, \mathbb{R}), \Xi=\mathbb{R}^{n}, \pi=$ the Weil representation.

$$
K(x, y)=\frac{c}{(2 \pi)^{\frac{n}{2}}} e^{-\sqrt{-1}\langle x, y\rangle}
$$

In this case $\mathcal{F}_{\Xi}$ is the Euclidean Fourier transform $\mathcal{F}_{\mathbb{R}^{n}}$ up to a phase factor $c$ with $|c|=1$, see $[\mathbf{2}]$.

2) $G=S O(p+1,2), \Xi$ is the light cone $(q=1$ in (2.1)), $\pi=$ the highest weight representation of the smallest Gelfand-Kirillov dimension and of the smallest $K$-type.

$$
K(x, y)=c \widetilde{J}_{\frac{p-3}{2}}(2 \sqrt{2\langle x, y\rangle})
$$


where $\widetilde{J}_{\lambda}(t):=\left(\frac{t}{2}\right)^{-\lambda} J_{\lambda}(t)$ is a renormalization of the J-Bessel function $([\mathbf{1 5}])$.

3.1. Mellin-Barnes type integral expression. In [17] we brought an idea of the Radon transform in the analysis of the unitary inversion operator $\mathcal{F}_{\Xi}$ for minimal representations. Recall that the Euclidean Fourier transform $\mathcal{F}_{\mathbb{R}^{n}}$ can be written as the composition of the one-dimensional Fourier transform and the Radon transform (plane wave decomposition). We can generalize this decomposition to the unitary inversion operator $\mathcal{F}_{\Xi}$ for the minimal representations and some small representations on $L^{2}(\Xi)$ given by Theorem 2.10, namely, there exists a distribution $\Phi(t)$ of one variable such that the distribution kernel $K(x, y)$ of $\mathcal{F}_{\Xi}$ is of the following form:

$$
K(x, y)=\Phi(\langle x, y\rangle)
$$

where $\langle$,$\rangle is some (natural) bilinear form of the ambient space V$. Thus Problem 2.5 reduces to find a formula of $\Phi(t)$.

ExAMPLE 3.2. In Example 3.1 (2) we have seen $\Phi(t)=c \widetilde{J}_{\frac{p-3}{2}}(2 \sqrt{2 t})$ when $G=O(p+1,2)$. Therefore, $\mathcal{F}_{\Xi}$ reduces to the Hankel transform composed with a 'Radon transform' on $\Xi$ in this case.

The formula of $\Phi(t)$ is more involved for $G=O(p+1, q+1)$ with $p, q \geq 2$ as the corresponding minimal representation is not a highest weight module and $\Phi(t)$ is not always locally integrable (see Question 3.4 below). An explicit formula of $\Phi(t)$ in this case can be given in terms of 'Bessel distributions' [17, Theorem 5.1.1]. Here we give an alternative expression of $\Phi(t)$, namely, by using a distribution-valued Mellin-Barnes integral.

We define a distribution of $t$ with meromorphic parameter $\lambda$ by

$$
b(\lambda, t):=\frac{\Gamma(-\lambda)}{\Gamma\left(\lambda+\frac{p+q}{2}-1\right)}(2 t)_{+}^{\lambda} .
$$

Here the Riesz distribution $(2 t)_{+}^{\lambda}$ is defined as a locally integrable function on $\mathbb{R}$ by

$$
(2 t)_{+}^{\lambda}= \begin{cases}(2 t)^{\lambda} & t>0 \\ 0 & t \leq 0\end{cases}
$$

for $\operatorname{Re} \lambda>-1$, and is extended as a distribution by the meromorphic continuation on $\lambda \in \mathbb{C}$. Let $m:=\frac{1}{2}(p+q-4)$, and $L_{m}$ be a contour starting at $\gamma-i \infty$, passes the real axis between $(-m-1,-m)$ and ends at $\gamma+i \infty$ when $\gamma>-1$. We define distributions $\Phi^{p, q}(t)$ by a distribution-valued Mellin-Barnes integral:

$$
\Phi^{p, q}(t):= \begin{cases}\int_{L_{0}} b(\lambda, t) d \lambda & (\text { Case A-1), } \\ \int_{L_{m}} b(\lambda, t) d \lambda & (\text { Case B-1), } \\ \int_{L_{m}}\left(\frac{b(\lambda, t)}{\tan \pi \lambda}+\frac{b(\lambda,-t)}{\sin \pi \lambda}\right) d \lambda & \text { (Case B-2), }\end{cases}
$$

according to the following three cases:

Case A-1. $\quad p=1$ or $q=1$,

Case B-1. $p, q>1$ and both odd,

Case B-2. $p, q>1$ and both even.

Then $\Phi^{p, q}(t)$ is independent of the choice of the contour and $\gamma$ under the above mentioned constraints.

Let $\langle$,$\rangle be the (positive definite) inner product on \mathbb{R}^{p, q}$. Then we have 
Theorem $3.3([\mathbf{1 7}, \S 6.2])$. For $G=O(p+1, q+1)$ with $p+q$ even, $\geq 4$, the kernel $K(x, y)$ of the unitary inversion $\mathcal{F}_{\Xi}$ is given by

$$
K(x, y)=c_{p, q} \Phi^{p, q}(\langle x, y\rangle)
$$

for some constant $c_{p, q}$.

3.2. Local integrability of the kernel. The kernel of the Euclidean Fourier transform $\mathcal{F}_{\mathbb{R}^{n}}$ is given by $e^{-i\langle x, \xi\rangle}$, which is locally integrable. We may ask to which extent this analytic feature remains to hold. To be more precise, let $\Phi(t)$ be the distribution on $\mathbb{R}$ as in (3.1). We ask

QUESTION 3.4. When is $\Phi(t)$ locally integrable?

For a Euclidean Jordan algebra $V$ we proved in $[\mathbf{9}]$ that $\Phi(t)$ is locally integrable. See (2.7) for the list of the corresponding conformal Lie algebras $\mathfrak{g}$.

For $G=O(p+1, q+1)$ with $p+q$ even $>2$, the Mellin-Barnes type integral formula (Theorem 3.3) leads to the following proposition (see [17, Theorem 6.2.1]):

Proposition 3.5. We have the identities modulo $L_{\text {loc }}^{1}\left(\mathbb{R}, r^{p+q-3} d r\right)$.

$$
\Phi^{p, q}(t) \equiv \begin{cases}0 & (\text { Case A-1) } \\ c_{1} \sum_{l=0}^{m-1} \frac{(-1)^{l}}{2^{l}(m-l-1) !} \delta^{(l)}(t) & (\text { Case B-1) } \\ c_{2} \sum_{l=0}^{m-1} \frac{l !}{2^{l}(m-l-1) !} t^{-l-1} & (\text { Case B-2) }\end{cases}
$$

for some nonzero constants $c_{1}, c_{2}$. Here $m=\frac{1}{2}(p+q-4)$.

Thus we have a complete answer to Question 3.4 in this case:

Corollary 3.6. $\Phi(t)$ is locally integrable if and only if $\mathfrak{g}=\mathfrak{o}(p+1,2), \mathfrak{o}(2, q+$ 1) or $\mathfrak{o}(3,3) \simeq \mathfrak{s l}(4, \mathbb{R})$.

We note that the minimal representations for $\mathfrak{g}=\mathfrak{o}(p+1,2)$ or $\mathfrak{o}(2, q+1)$ are highest (or lowest) weight modules, whereas minimal representations do not make sense for $\mathfrak{g}=\mathfrak{o}(3,3)$ which is isomorphic to $\mathfrak{s l}(4, \mathbb{R})$. (Recall that the Joseph ideal is defined when $\mathfrak{g}_{\mathbb{C}}$ is not of type $A$.)

The delicate answer indicated in Corollary 3.6 is closely related to the regularity of the 'Radon transform' on $\Xi$. To be more precise, the Radon transform on $\Xi$ is defined as the integral over the codimension-one submanifold

$$
\langle x, y\rangle=t \quad \text { in } \Xi .
$$

which collapses when $t=0$. Accordingly, the Radon transform

$$
(\mathcal{R} u)(x, t)=\int_{\Xi} u(y) \delta(\langle x, y\rangle-t) d y
$$

has a better regularity as $t$ tends to 0 . (On the other hand, the asymptotic behavior as $|t| \rightarrow \infty$ is similar to the Euclidean case.) The singular part of $\Phi^{p, q}(t)$ in Proposition 3.5 fits well with the behavior of $\mathcal{R} u(x, t)$ as $t$ tends to 0 .

Question 3.4 is open for minimal representations without highest weights except for the case $G=O(p+1, q+1)$. 


\section{Fourth order differential equations}

4.1. Gaussian kernel and minimal $K$-type. The Euclidean Fourier transform $\mathcal{F}_{\mathbb{R}^{n}}$ is of order four, and therefore its eigenvalues in $L^{2}\left(\mathbb{R}^{n}\right)$ are among $\{ \pm 1, \pm \sqrt{-1}\}$. An important eigenfunction with eigenvalue 1 is the Gaussian kernel $e^{-\frac{1}{2}\|x\|^{2}}$, namely,

$$
\mathcal{F}_{\mathbb{R}^{n}}\left(e^{-\frac{1}{2}\|x\|^{2}}\right)=e^{-\frac{1}{2}\|x\|^{2}} .
$$

Thus the Gaussian kernel $e^{-\frac{1}{2}\|x\|^{2}}$ is a square integrable function on $\mathbb{R}^{n}$ satisfying the following property:

$$
\mathcal{F}_{\mathbb{R}^{n}} f=f \quad \text { and } \quad f \text { is } O(n) \text {-invariant. }
$$

Next let $p \geq q \geq 1, p+q$ even, and we consider the isotropic cone $\Xi$ in $\mathbb{R}^{p, q}$ as in (2.1). The unitary inversion operator $\mathcal{F}_{\Xi}$ of the minimal representation $\pi$ of $O(p+1, q+1)$ is of order two because $\mathcal{F}_{\Xi}=\pi(w)$ and $w^{2}=I_{p+q}($ see $(2.4))$, and therefore its eigenvalues in $L^{2}(\Xi)$ are either 1 or -1 . An important eigenfunction of $\mathcal{F}_{\Xi}$ is $\widetilde{K}_{\frac{1}{2}(q-2)}(2\|x\|)$ where $\widetilde{K}_{\lambda}(t):=\left(\frac{t}{2}\right)^{-\lambda} K_{\lambda}(t)$ is a renormalization of the KBessel function. This is a square integrable function on $\Xi$ satisfying the following property:

$$
\mathcal{F}_{\Xi} f= \pm f \quad \text { and } \quad f \text { is } O(p) \times O(q) \text {-invariant }
$$

To be more precise,

$$
\mathcal{F}_{\Xi f}=\left\{\begin{array}{lll}
f & \text { if } p-q \equiv 0 & \bmod 4, \\
-f & \text { if } p-q \equiv 2 & \bmod 4 .
\end{array}\right.
$$

ExAmPLE 4.1. For $p=3$ and $q=1$, we have

$$
\mathcal{F}_{\Xi}\left(e^{-2\|x\|}\right)=-e^{-2\|x\|}
$$

because

$$
\widetilde{K}_{-\frac{1}{2}}(t)=\frac{\sqrt{\pi}}{2} e^{-t}
$$

The function $e^{-2\|x\|}$ arises as the wave function for the hydrogen atom with the lowest energy.

From the view point of representation theory, the Gaussian kernel $e^{-\frac{1}{2}\|x\|^{2}}$ generates the minimal $K$-type of the Weil representation of $M p(n, \mathbb{R})$, whereas the function $\widetilde{K}_{\frac{1}{2}(q-2)}(2\|x\|)$ generates that of the minimal representation of $O(p+1, q+$ 1) realized in $L^{2}(\Xi)$.

4.2. The Mano polynomial. We recall a classical fact that the Hermite polynomials form an orthogonal basis for the radial part of the Schrödinger model $L^{2}\left(\mathbb{R}^{n}\right)$ of the Weil representation e.g. $[\mathbf{2}, \mathbf{1 0}]$, whereas the Laguerre polynomials arise in the minimal representation of the conformal group $S O(4,2)$. The bottom of the series correspond to what we have discussed in Section 4.1.

We notice that these two minimal representations are quite special, namely, they are highest weight modules. However, for more general reductive groups, minimal representations are not always highest weight modules, and we need new 'orthogonal polynomials' and 'special functions' to describe a natural basis of functions satisfying (4.2) or alike. 
For $\mu \in \mathbb{C} \backslash\{-1,-2,-3, \cdots\}$ and $\ell \in \mathbb{N}$, the Mano polynomials $\left\{M_{j}^{\mu, \ell}(x)\right\}_{j \in \mathbb{N}}$ are defined by

$$
M_{j}^{\mu, \ell}(x):=\left.\frac{\Gamma(j+\mu+1)}{j ! 2^{\mu} \Gamma\left(j+\frac{\mu+1}{2}\right)} \frac{\partial^{j}}{\partial t^{j}}\right|_{t=0} G^{\mu, \ell}(t, x),
$$

where the generating function $G^{\mu, l}(t, x)$ is given by

$$
G^{\mu, \ell}(t, x):=\frac{\left(\frac{x}{2}\right)^{2 \ell+1} e^{\frac{x}{2}}}{(1-t)^{\ell+\frac{\mu+3}{2}}} \widetilde{I}_{\frac{\mu}{2}}\left(\frac{t x}{2(1-t)}\right) \widetilde{K}_{\ell+\frac{1}{2}}\left(\frac{x}{2(1-t)}\right) .
$$

Here $\widetilde{I}_{\alpha}(z):=\left(\frac{z}{2}\right)^{-\alpha} I_{\alpha}(z)$ and $\widetilde{K}_{\alpha}(z)=\left(\frac{z}{2}\right)^{-\alpha} K_{\alpha}(z)$ denote the renormalized $I$ and $K$-Bessel functions. With this normalization, the polynomial $M_{j}^{\mu, \ell}(x)$ is of the following top term:

$$
M_{j}^{\mu, \ell}(x)=\frac{(-1)^{j}}{j !} x^{j+\ell}+\text { lower order terms. }
$$

EXAMPLE 4.2 (Special values of the Mano polynomial).

(1) The bottom of the series with $j=0$ is related with the $K$-Bessel functions with half-integer parameter:

$$
M_{0}^{\mu, \ell}(x)=\pi^{-\frac{1}{2}} z^{2 l+1} e^{z} \widetilde{K}_{\ell+\frac{1}{2}}(z)\left(=\sum_{k=0}^{\ell} \frac{(2 \ell-k) !}{k !(\ell-k) !} x^{k}\right) .
$$

(2) The polynomials $M_{j}^{\mu, \ell}(x)$ for $\ell=0$ reduce to the Laguerre polynomials

$$
M_{j}^{\mu, 0}(x)=L_{j}^{\mu}(x)\left(=\frac{\Gamma(n+\nu+1)}{n !} \sum_{k=0}^{n}(-1)^{k}\left(\begin{array}{l}
n \\
k
\end{array}\right) \frac{x^{k}}{\Gamma(k+\nu+1)}\right) .
$$

(3) The function $M_{j}^{\mu, \ell}(x)$ is not a polynomial when $\ell \notin \mathbb{N}$, but it is convenient to include the negative integer case. In particular, for $\ell=-1$, it follows from [6, Corollary 5.3] and [7, Lemma 3.2] that $M_{j}^{\mu,-1}(x)$ is essentially the Laguerre polynomial:

$$
x M_{j}^{\mu,-1}(x)=L_{j}^{\mu}(x) \quad(j \in \mathbb{N}) .
$$

Many of the classical orthogonal polynomials are obtained as eigenfunctions of self-adjoint differential operators of second-order, but the Mano polynomials $M_{j}^{\mu, \ell}(x)$ are obtained as those of fourth-order. Indeed this is a requirement from representation theory because the Casimir operator (for a compact subgroup) acts as a fourth-order differential operator on $\Xi$. To see this we may recall that the Lie algebra $\overline{\mathfrak{n}}$ acts as a second order differential operator (e.g. the fundamental differential operator $R_{j}$ in $(2.3)$ ).

We begin with a second order differential operator on $\mathbb{R}$

$$
\mathcal{R}_{\mu, \ell}:=\left(x \frac{d}{d x}+\mu-2 \ell-1-\frac{x}{2}\right)\left(x \frac{d}{d x}+\mu-\frac{x}{2}\right)-\left(\frac{x}{2}\right)^{2},
$$

and introduce a fourth order differential operator

$$
\mathcal{P}_{\mu, \ell}:=\frac{1}{x^{2}} \mathcal{R}_{\mu, \ell} \mathcal{R}_{0, \ell} .
$$


Proposition $4.3([\mathbf{7}])$. (1) (Differential equation) The Mano polynomial $M_{j}^{\mu, \ell}(x)$ is an eigenfunction of $\mathcal{P}_{\mu, \ell}$ :

$$
\mathcal{P}_{\mu, \ell} u=j(j+\mu+1) u .
$$

(2) (Completeness) If $\mu \geq 2 \ell+1$ is an odd integer, then $\left\{M_{j}^{\mu, \ell}\right\}_{j \in \mathbb{N}}$ forms an orthogonal basis of $L^{2}\left(\mathbb{R}_{+}, x^{\mu-2 \ell} e^{-x} d x\right)$.

There are two proofs of an explicit formula for the $L^{2}$-norm of $M_{j}^{\mu, \ell}(x)$, see [7, Theorem 2.4] and [18, Corollary 4.1].

4.3. $K$-finite vectors for minimal representations. We discuss a relationship between the Mano polynomials and minimal representations. Let $\Xi$ be the isotropic cone in $\mathbb{R}^{p, q}$ as in (2.1) with $p+q \geq 4$ and even.

We recall that the space of $k$ th spherical harmonics

$$
\mathcal{H}^{k}\left(\mathbb{R}^{n}\right):=\left\{\varphi \in C^{\infty}\left(S^{n-1}\right): \Delta_{S^{n-1}} \varphi=-k(k+n-2) \varphi\right\}
$$

is a finite dimensional vector space, on which the orthogonal group $O(n)$ acts irreducibly by rotations. Then we have an irreducible representation of $K:=$ $O(p+1) \times O(q+1)$ on

$$
V^{j}:=\mathcal{H}^{j}\left(\mathbb{R}^{p+1}\right) \otimes \mathcal{H}^{j+\frac{p-q}{2}}\left(\mathbb{R}^{q+1}\right), \quad j=0,1,2, \cdots .
$$

Let $L^{2}(\Xi)_{K}$ be the underlying $(\mathfrak{g}, K)$-module of the minimal representation $L^{2}(\Xi)$ of $G=O(p+1, q+1)$. Then by $[\mathbf{1 9}], L^{2}(\Xi)_{K}$ is isomorphic to the multiplicity-free sum $\oplus_{j=0}^{\infty} V^{j}$ as $K$-modules. We write $L^{2}(\Xi)_{j}$ for the corresponding $K$-irreducible subspace of $L^{2}(\Xi)$.

With the notation of Section $2.2, K \cap P \simeq O(p) \times O(q) \times \mathbb{Z}_{2}$. We set $M:=$ $O(p) \times O(q)$. The representation of the whole group $G$ (or even the maximal compact subgroup $K$ ) on $L^{2}(\Xi)$ does not come from the geometric action of $G$ on $\Xi$, but the action of the subgroup $M$ is given by rotation in the argument and the bipolar coordinates (2.2) respect the $M$-action. Correspondingly, it is not straightforward to find explicit $K$-finite vectors in $L^{2}(\Xi)$, whereas the $M$-invariant functions only depend on the radial parameter $r \in \mathbb{R}_{+}$. We identify the space $L^{2}(\Xi)^{M}$ of $M$ invariants in $L^{2}(\Xi)$ with $L^{2}\left(\mathbb{R}_{+},(1 / 2) r^{p+q-3} d r\right)$ as we saw in Section 2.1.

Proposition 4.4 ([6, Section 8]). Let $G=O(2 m, 2 n)$. For every $j \in \mathbb{N}$, the subspace $L^{2}(\Xi)_{j} \cap L^{2}(\Xi)^{M}$ is one-dimensional and given by the Mano polynomials:

$$
u_{j}^{m, n}(x):=x^{-2 n+3} e^{-x} M_{j}^{2 m-3,2 n-3}(x) .
$$

Here $x=2 r$ in the bipolar coordinates (2.2) of $\Xi$.

Therefore the functions $\left\{u_{j}^{m, n}(2 r)\right\}_{j \in \mathbb{N}}$ give a basis of functions $f$ satisfying (4.2). To be more precise, we have

$$
\mathcal{F}_{\Xi} u_{j}^{m, n}=\left\{\begin{array}{lll}
u_{j}^{m, n} & \text { if } n-m \equiv j \quad \bmod 2, \\
-u_{j}^{m, n} & \text { if } n-m \neq \equiv j \quad \bmod 2 .
\end{array}\right.
$$

EXAMPLE 4.5. 1) The bottom parameter $j=0$ explains that $\widetilde{K}_{\frac{1}{2}(q-2)}(2 r)$ generates the minimal $K$-type (Section 4.1 ).

2) The case $n=1$ recovers the classical fact that the Laguerre polynomials generate every $K$-finite vectors for the minimal representations of $S O(2 m, 2)$. 
The following remarkable observation is a consequence of Example 4.2 (3) and Proposition 4.4:

Observation 4.6. Let $G=O(2 m, 4)$ with $m \geq 2$. Then any $K$-type of $\pi$ is given by using Laguerre polynomials.

We notice that the minimal representations of $O(2 m, 4)$ are not highest weight modules. It would be interesting to find a different proof for Observation 4.6, possibly in connection with other areas of mathematics.

In contrast to Proposition 4.4 for $G=O(2 m, 2 n), K$-finite vectors in $L^{2}(\Xi)$ cannot be expressed by elementary functions for $G=O(2 m+1,2 n+1)$. For this we introduced in $[\mathbf{6}]$ a family of real analytic functions $\left\{\Lambda_{j}^{\mu, \nu}(x)\right\}_{j \in \mathbb{N}}$ on $\mathbb{R}_{+}$by the generating function:

$$
\sum_{j=0}^{\infty} t^{j} \Lambda_{j}^{\mu, \nu}(x)=\frac{1}{(1-t)^{\frac{\mu+\nu+2}{2}}} \widetilde{I}_{\frac{\mu}{2}}\left(\frac{t x}{1-t}\right) \widetilde{K}_{\frac{\nu}{2}}\left(\frac{x}{1-t}\right) .
$$

Then we have the following Proposition:

Proposition 4.7 ([6, Corollary 8.2]). Let $G=O(p+1, q+1)$ with $p+q$ even, $>2$. Then

for all $j \in \mathbb{N}$.

$$
L^{2}(\Xi)_{j} \cap L^{2}(\xi)^{M}=\mathbb{C} \Lambda_{j}^{p-2, q-2}(2 r)
$$

Corollary 4.8. For $\mu \geq \nu \geq-1, \mu, \nu \in \mathbb{Z}$ with $\mu \equiv \nu \bmod 2$ and $(\mu, \nu) \neq$ $(-1,-1),\left\{\Lambda_{j}^{\mu, \nu}(x)\right\}_{j \in \mathbb{N}}$ forms an orthogonal basis in $L^{2}\left(\mathbb{R}_{+}, x^{\mu+\nu+1} d x\right)$.

REMARK 4.9. The function $\Lambda_{j}^{\mu, \nu}$ can be expressed by elementary functions when $\nu \in 2 \mathbb{Z}+1$ :

$$
\Lambda_{j}^{\mu, 2 \ell+1}(x)=\frac{2^{\mu} \Gamma\left(j+\frac{\mu+1}{2}\right)}{\Gamma(j+\mu+1)} x^{-2 \ell-1} e^{-x} M_{j}^{\mu, \ell}(2 x) .
$$

Thus Proposition 4.7 includes Proposition 4.4 as a special case.

REMARK 4.10. The indefinite orthogonal group $O(p, q)$ has two parameters $p$ and $q$, and the corresponding special functions are expected to be the most general. In fact, for other minimal representations and some small representations given in Theorem 2.10, analogous results of Proposition 4.7 remain true by a specific choice of the parameters $\mu$ and $\nu$.

\section{References}

1. S. Ben Saïd, T. Kobayashi, and B. Ørsted, Laguerre semigroup and Dunkl operators, Composition Math. 148 (2012), pp. 1265-1336.

2. G. B. Folland, Harmonic analysis in phase space, Annals of Mathematics Studies 122, Princeton University Press, Princeton, NJ, 1989.

3. I. Frenkel and M. Libine, Quaternionic analysis, representation theory and physics, Adv. Math. 218 (2008), 1806-1877.

4. I. Frenkel and M. Libine, Split quaternionic analysis and separation of the series for $S L(2, \mathbb{R})$ and $S L(2, \mathbb{C}) / S L(2, \mathbb{R})$. Adv. Math. 228 (2011), 678-763.

5. I. Frenkel and M. Libine, Quaternionic Analysis and the Schrdinger Model for the Minimal Representation of $O(3,3)$, International Mathematics Research Notices, 2012-21, (2012), pp. 4904-4923.

6. J. Hilgert, T. Kobayashi, G. Mano, and J. Möllers, Special functions associated to a certain fourth order differential operator, The Ramanujan Journal 26 (2011), 1-34. 
7. J. Hilgert, T. Kobayashi, G. Mano, and J. Möllers, Orthogonal polynomials associated to a certain fourth order differential equation, The Ramanujan Journal 26 (2011), 295-310.

8. J. Hilgert, T. Kobayashi and J. Möllers, Minimal representations via Bessel operators, 72 pp. to appear in Jour. Math. Soc. Japan (cf. arXiv:1106.3621 ).

9. J. Hilgert, T. Kobayashi, J. Möllers, and B. Ørsted, Segal-Bargmann transform and Fock space realization for minimal holomorphic representations, J. Funct. Anal. 263 (2012), 34923563 (cf. arXiv:1203.5462 )

10. R. Howe, The oscillator semigroup, Proc. Sympos. Pure Math. 48, Amer. Math. Soc., 1988, 61-132.

11. A. Joseph, The minimal orbit in a simple lie algebra and its associated maximal ideal, Ann. Scient. Ec. Norm. Sup. 9 (1976), 1-30.

12. T. Kobayashi, Conformal geometry and global solutions to the Yamabe equations on classical pseudo-Riemannian manifolds, Rend. Circ. Mat. Palermo (2) Suppl. 71, (2003), pp. 15-40.

13. T. Kobayashi, Algebraic analysis of minimal representations, Publ. Res. Inst. Math. Sci. 47 (2011), 585-611, Special issue in commemoration of the golden jubilee of algebraic analysis.

14. T. Kobayashi, Varna lecture on $L^{2}$-analysis of minimal representations, In: Lie Theory and Its Applications in Physics, IXth International Workshop 2011 held at Varna (ed., V. Dobrev), Springer Proceedings in Mathematics \& Statistics, vol. 36, Springer, 2013, pp. 77-93.

15. T. Kobayashi and G. Mano, Integral formulas for the minimal representation of $\mathrm{O}(p, 2)$, Acta Appl. Math. 86 (2005), 103-113.

16. T. Kobayashi and G. Mano, The inversion formula and holomorphic extension of the minimal representation of the conformal group, In: Harmonic Analysis, Group Representations, Automorphic Forms and Invariant Theory: In honor of Roger E. Howe, Singapore University Press and World Scientific Publishing, 2007, pp. 159-223 (available at math.RT/0607007).

17. T. Kobayashi and G. Mano, The Schrödinger model for the minimal representation of the indefinite orthogonal group $O(p, q)$, Mem. Amer. Math. Soc. (2011), 212, no. 1000 , vi+132 pp. (available at arXiv:0712.1769)

18. T. Kobayashi and J. Möllers, An integral formula for $L^{2}$-eigenfunctions of a fourth order Bessel-type differential operator, Integral Transforms and Special Functions 22 (2011), 521531.

19. T. Kobayashi and B. Ørsted, Analysis on the minimal representation of $\mathrm{O}(p, q)$. I. - Realization and conformal geometry, II. - Branching laws, III. - Ultra-hyperbolic equations on $\mathbb{R}^{p-1, q-1}$, , Adv. Math. 180 (2003), 486-512; 513-550; 551-595.

20. T. Kobayashi, B. Ørsted, and M. Pevzner, Geometric analysis on small unitary representations of $G L(N, \mathbb{R})$, J. Funct. Analy. 260 (2011), 1682-1720.

21. B. Kostant, The vanishing of scalar curvature and the minimal representation of $\mathrm{SO}(4,4)$, Operator algebras, unitary representations, enveloping algebras, and invariant theory (A. Connes, M. Duflo, A. Joseph, and R. Rentschler, eds.), Progr. Math., 92, Birkhäuser, Boston, 1990, pp. 85-124.

22. A. Kowata and M. Moriwaki, Invariant differential operators on the Schrödinger model for the minimal representation of the conformal group, J. Math. Sci. Univ. Tokyo, 18 (2011), $355-395$.

23. D. A. Vogan, Jr., Singular unitary representations, Noncommutative harmonic analysis and Lie groups (Marseille, 1980), Lecture Notes in Math., vol. 880, Springer, Berlin, 1981, pp. 506535.

Kavli IPMU and Graduate School of Mathematical Sciences, The University of Tokyo, 3-8-1 Komaba, Meguro, TOKyo, 153-8914, Japan.

E-mail address: toshi@ms.u-tokyo.ac.jp 